\title{
Diagnosis of congenital pericardial defects, including a pathognomic sign for dangerous apical ventricular herniation, on magnetic resonance imaging
}

Ingmar Gassner, Werner Judmaier, Christoph Fink, Manfred Lener, Ferdinand Waldenberger, Helmut Scharfetter, Ignaz Hammerer

\begin{abstract}
Objective-To establish criteria for the accurate diagnosis of different forms of left sided pericardial defects on magnetic resonance imaging. Early detection of a partial apical defect is essential as it is potentially fatal.

Design-Examination of four children with congenital pericardial defects by magnetic resonance imaging, the results being compared with the features on conventional chest radiography and echocardiography and with published data.

Results-Magnetic resonance imaging improved the ability to diagnose and distinguish between complete and partial left sided pericardial defects. A deep myocardial crease was visualised in a patient with apical pericardial defect, indicating the risk of a life threatening ventricular strangulation. A prominent left atrial appendage was, in contrast to many reports, not a reliable sign for partial left sided pericardial defect.

Conclusions-The various forms of congenital left sided pericardial defects cannot reliably be diagnosed in plain chest radiographs or on echocardiography. Their diagnosis and the distinction between partial and complete defects, however, is of clinical importance and can be accomplished more confidently by magnetic resonance imaging.
\end{abstract}

(Br Heart f 1995;74:60-66)

Keywords: congenital pericardial defects; apical ventricular herniation; magnetic resonance imaging

The radiological features in chest radiographs of complete and partial pericardial defects have been described. ${ }^{1-5}$ Whereas a complete left sided pericardial defect has little importance for the wellbeing of the patient, a partial left sided pericardial defect can indicate a life threatening condition. The features in chest radiographs have, however, proved to be sometimes absent or misleading. ${ }^{2}$

The purpose of this study was to determine whether magnetic resonance imaging can be used to diagnose left sided pericardial defects and to identify the different types.
Patients and methods

We examined four children with congenital pericardial defects by magnetic resonance imaging, comparing the results with those on conventional chest radiography and echocardiography and with published data.

Magnetic resonance imaging was performed on a $1.5 \mathrm{~T}$ superconducting magnet (Magnetom, Siemens, Erlangen, Germany). Imaging variables included an acquisition matrix of $128 \times 256$ to $192 \times 512$, a field of view of $385 \mathrm{~cm}$, four excitations, and a slice thickness of 5-8 mm with a gap of $0-2 \mathrm{~mm}$ between sections. Electrocardiographically triggered coronal and axial $\mathrm{T} 1$ weighted spin echo images were obtained with an echo time of $15 \mathrm{~ms}$ or $22 \mathrm{~ms}$ and a variable repetition time between $580 \mathrm{~ms}$ and $1100 \mathrm{~ms}$, depending on the child's RR interval.

\section{Results}

CASE 1

A boy presented at the age of 3 years with a murmur characteristic of persistent ductus arteriosus. A conventional chest radiograph showed a somewhat enlarged heart but no displacement to the left. Pulmonary vascularity was increased. A slight spherical bulge over the left hilum was interpreted as ductal diverticulum. At thoracotomy the duct had a diameter of about $5 \mathrm{~mm}$ without evidence of a ductal diverticulum. The main pulmonary artery and left pulmonary artery were dilated. A previously unsuspected complete left sided pericardial defect was discovered.

Only at the age of 13 years did a chest $x$ ray film show all the criteria of a complete left sided pericardial defect, including leftward displacement of the heart. At this age echocardiography and magnetic resonance imaging were performed. The findings are summarised in the table.

CASE 2

A boy had a chest radiography at the age of 6 months because of a respiratory tract infection. At fluoroscopy a remarkable bulge in the left hilar region was identified as a strongly pulsating left atrial appendage. We assumed that herniation had occurred through a partial left sided pericardial defect. \\ Children's Hospita
University of
Innsbruck, Austr
I Gassner
Institute of Magn
Resonance Imagi
and Spectroscopy
University of
Innsbruck
W Judmaier
M Lener
Department of
Paediatric Cardi
}


Summary of radiological and surgical findings in four patients studied

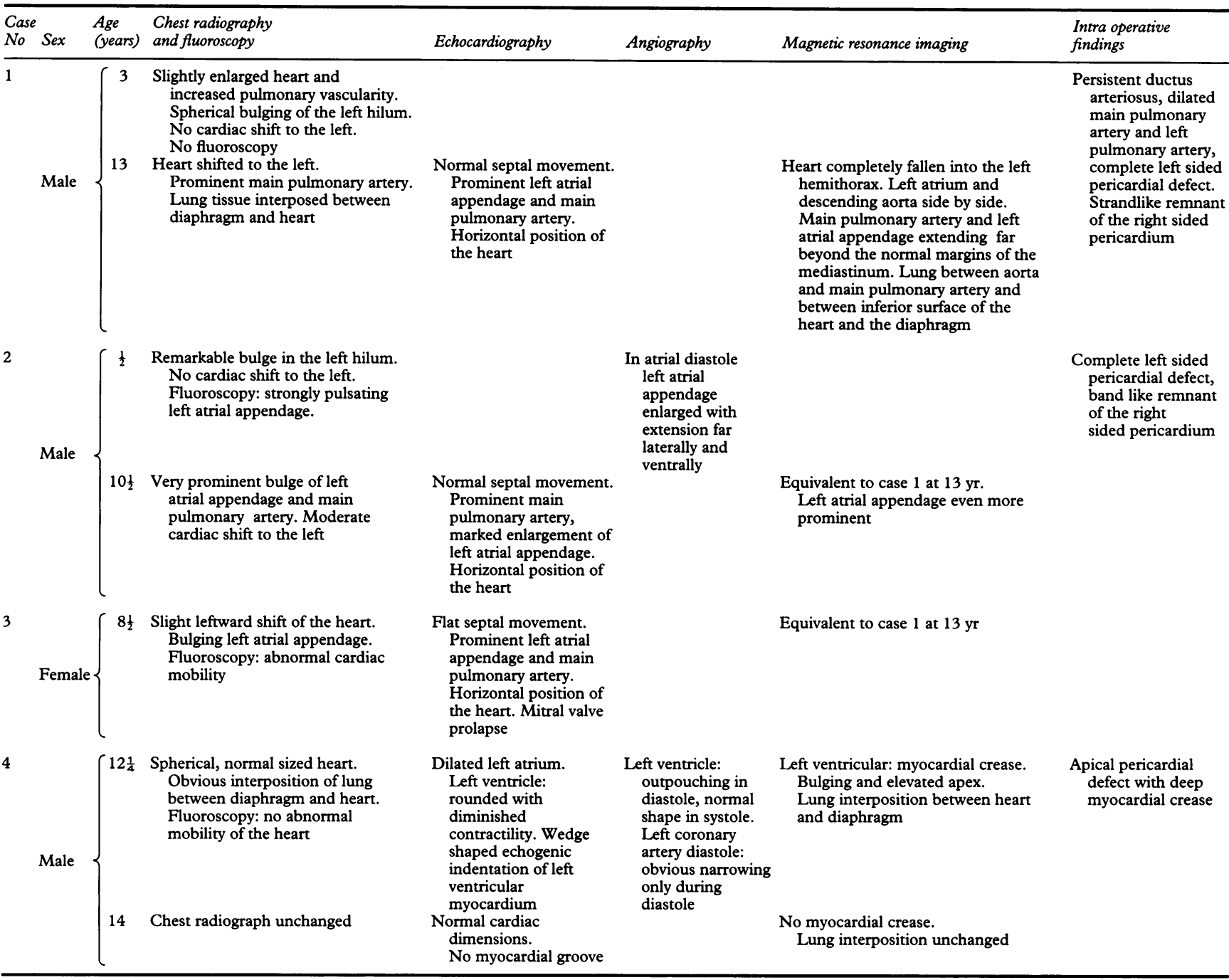

Figure 1 Case 2. Chest radiograph when patient was aged $10 \frac{1}{2}$ years

showing prominence of the left atrial appendage and leftward displacement of the heart.
An angiocardiogram showed further evidence for this assumption: in atrial diastole the enlarged atrial appendage extended far laterally and ventrally. Because of the reported risks of herniation with subsequent strangulation of the left ventricle he was sent for thoracotomy. Intraoperatively a complete left sided pericardial defect was found, but this needed no correction.

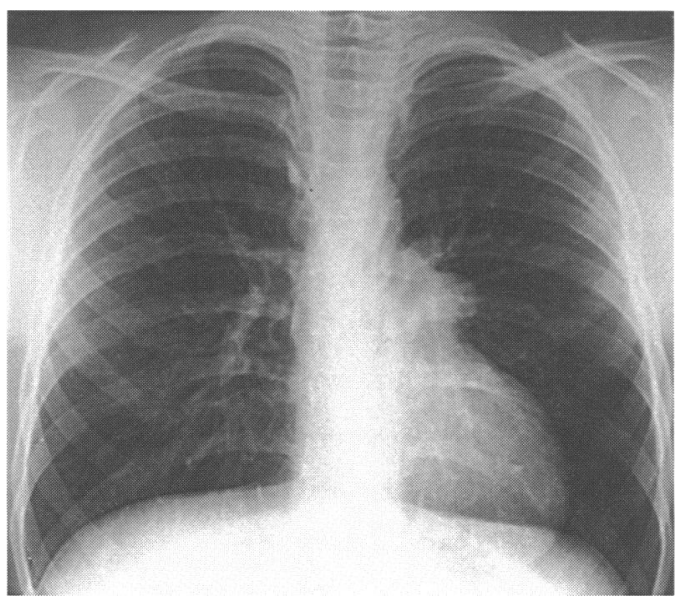

A follow up chest $x$ ray film 10 years later showed an extremely prominent left atrial appendage and a moderate leftward shift of the heart (figure 1). Echocardiography and magnetic resonance imaging were performed (figures 2-4; table).

\section{CASE 3}

A girl aged 8 years and 6 months who had been free of symptoms presented with a slight pectus excavatum and a heart murmur caused by an echocardiographically proved mitral valve prolapse. In a chest $x$ ray film the middle shadow was somewhat dislocated to the left, and immediately below the pulmonary artery there was a bulge corresponding to the left atrial appendage on fluoroscopy. When she was moved to the left lateral decubitus position the heart was displaced and abutted the left thoracic wall, which was strongly suggestive of a complete left sided pericardial defect. This finding was even more apparent on magnetic resonance imaging.

CASE 4

A boy aged 12 years and 3 months had a history of decreasing physical fitness over nine months. After swimming and drinking a large 
Figure 2 Case 2. Coronal magnetic resonance tomogram. Because of the left and dorsal displacement of the heart the descending aorta and the left atrium abut each other (arrowheads). pericardial layer allows the left atrial appendage to expand (arrow). The triangular signal loss below the inferior surface of the heart is caused by lung tissue taking the place of the pericardiodiaphragmatic The absence of the junction (star).
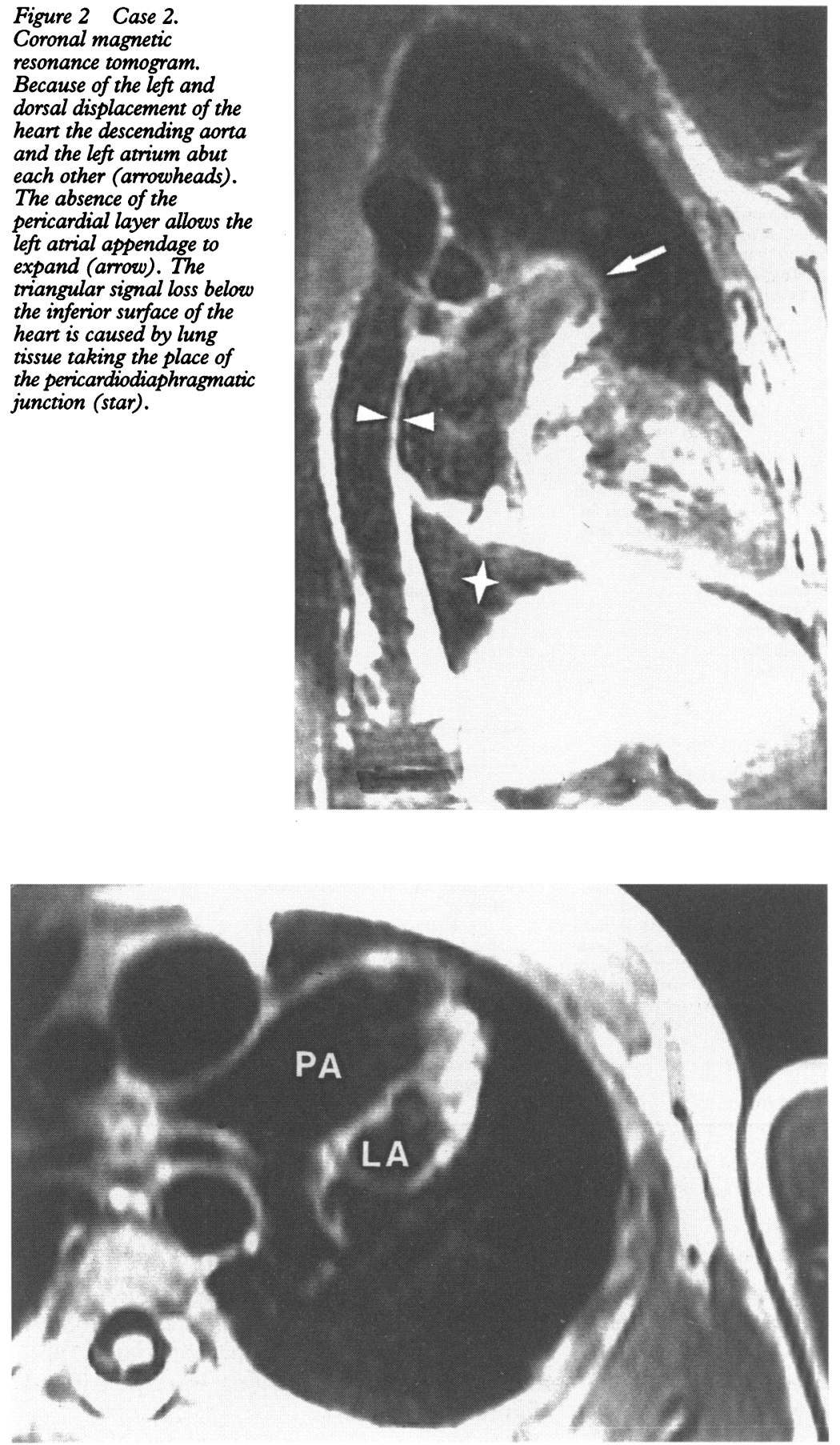

Figure 3 Case 2. Axial magnetic resonance tomogram at the level of the great vessels. Main pulmonary artery (PA) and left atrial appendage ( $L A)$ are protruding far to the left. Lung is interposed between the aorta and the pulmonary artery.

Figure 4 Case 2. Axial magnetic resonance tomogram at the level of the ventricles. In the supine position the heart completely fell into the left hemithorax. The left atrium is wrapped over the aorta.

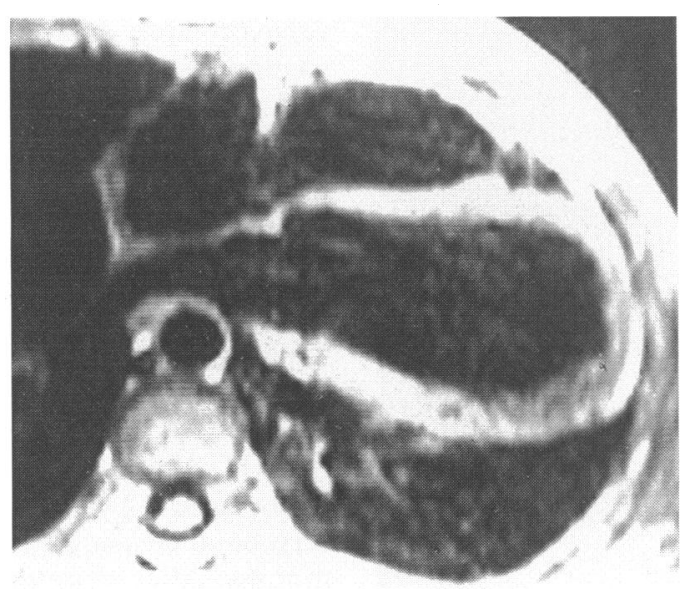

amount of a carbonated soft drink he suffered severe retrosternal stinging pain, which extended to the left shoulder and the left arm. In addition, he sweated profusely, felt nauseous, and repeatedly vomited. The pain worsened on deep inspiration.

An electrocardiogram showed signs of an anterolateral myocardial infarction. Activities of the heart muscle specific isoenzymes of creatine kinase and lactate dehydrogenase were raised.

In a chest $x$ ray film the normal sized heart was almost spherical, with interposition of lung between the diaphragm and the heart (figure 5). Fluoroscopy did not show an abnormal postural mobility of the heart. On echocardiography the left ventricle was remarkably round, with an apparent hypokinesia of the anterior wall. Apical to the sulcus atrioventricularis there was a wedge shaped area-with increased echogenicity indenting the left ventricular myocardium (figure 6).

In subsequent magnetic resonance tomograms we noted a striking elevation of the apex of the heart, well away from the surface of the diaphragm (figure 7), and, especially on axial images, a well defined indentation of the outer margin of the left ventricle. This pleat was most pronounced at the surface of the heart near the diaphragm and extended cranially along both the posterior and anterior wall. Abutting this sulcus was a collar-like accumulation of pericardial fat (figure 8).

Although these findings suggested ventricular herniation through an apical partial left sided pericardial defect, cineangiography was performed to ascertain the diagnosis. It showed an outpouching of the left ventricle, from its base to the apex, which was only visible in diastole (figure 9). In systole the shape of the left ventricle seemed to be almost normal. The left anterior descending coronary artery showed a subtotal occlusion in ventricular diastole shortly after its origin from the aorta,

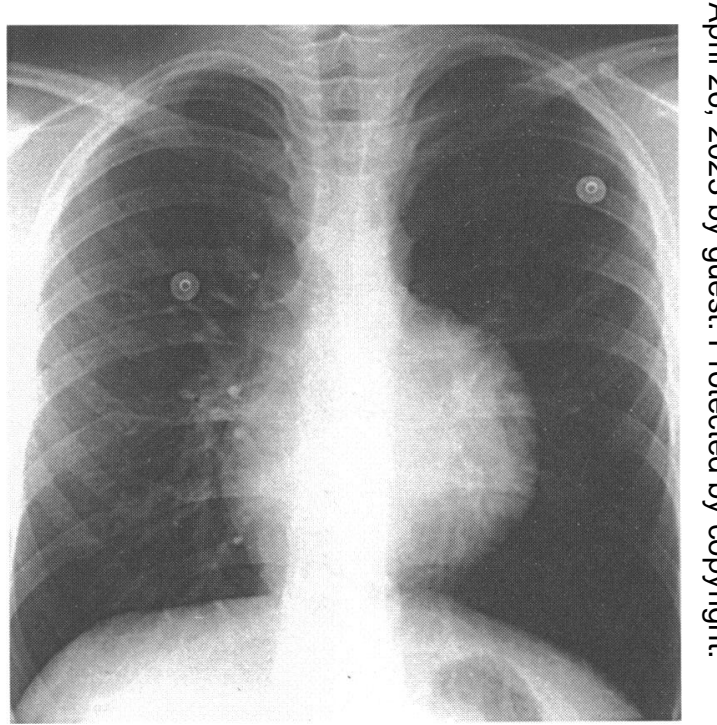

Figure 5 Case 4. Chest radiograph when patient was aged 12 years showing a spherical heart and interposition of lung between inferior cardiac surface and left hemidiaphragm. 


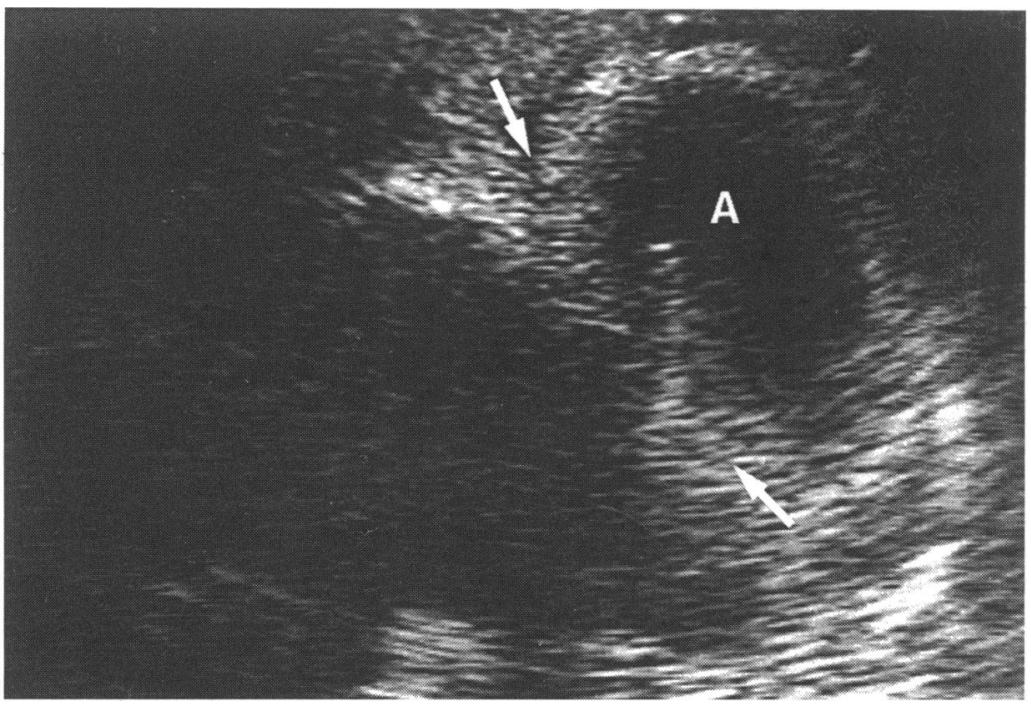

Figure 6 Case 4. Echocardiogram (subxiphoidal approach) showing a pronounced groove (arrows) in the diaphragmatic surface of the myocardium. $A$, apex of the left ventricle.

Figure 7 Case 4. Coronal magnetic resonance tomogram ( $T 1$ weighted spin-echo) showing lung tissue separating the diaphragm and the heart. A groove is noted in the diaphragmatic portion of the left ventricular myocardium (arrow).

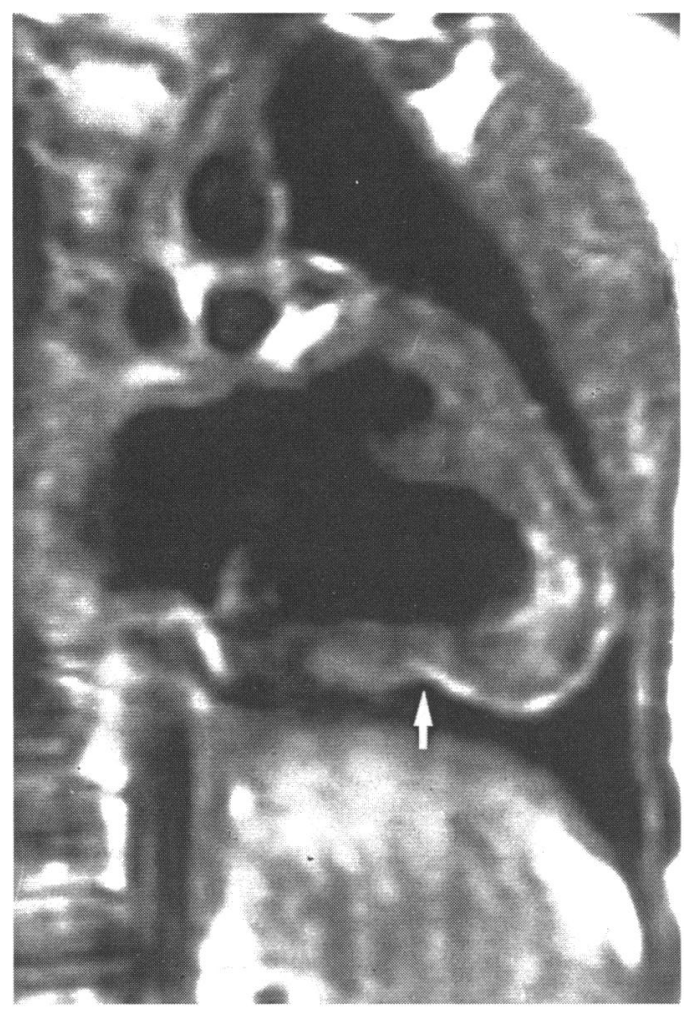

with a normal diameter in systole (figure 10).

The diagnosis of a partial left sided pericardial defect with herniation of the left ventricle and diastolic compression of the left coronary artery due to the free edge of the pericardium was established. Intraoperatively the left sided pericardium proved to be absent in the apical region, thus the apex and the anterior and posterior wall lay outside the pericardial sac. Along the free rim of the defect there was a deep indentation of the myocardium with subepicardial haemorrhage. The left phrenic nerve was displaced ventrally and lay within the free edge of the pericardium. The pericardial gap was widened surgically and the phrenic nerve transposed dorsally.

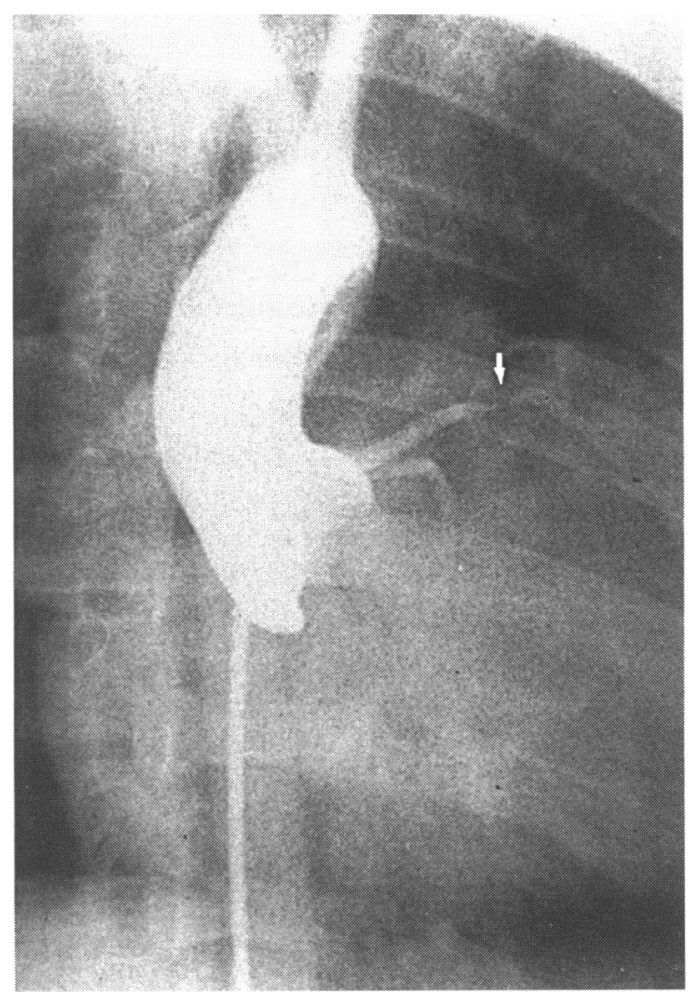

Figure 10 Case 4. Aortic root injection. Narrowing of the lumen of the left anterior descending coronary artery is seen only in ventricular diastole (arrow).

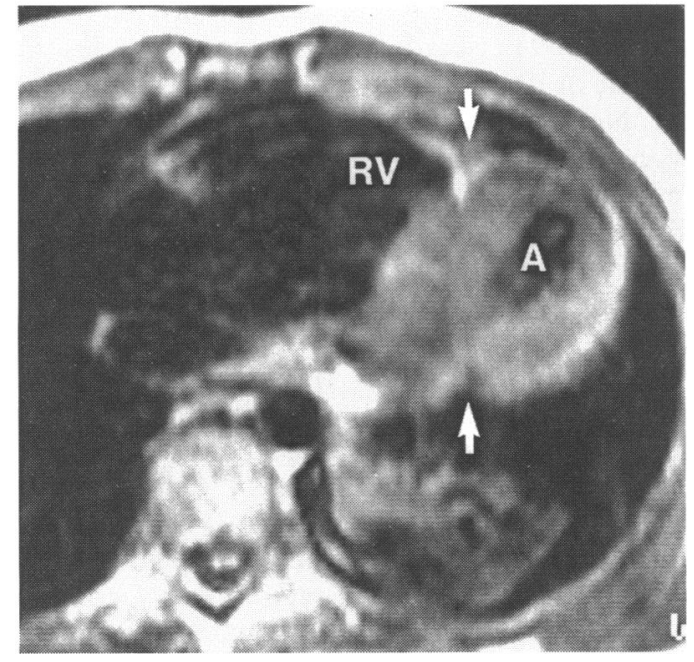

Figure 8 Case 4. Axial magnetic resonance tomogram at the apical level of the heart showing section comparable with that in figure 6, and myocardial groove (arrows). RV, right ventricle; $A$, apex of the left ventricle. 
Two years after surgery the patient was symptom free and the myocardial indentation was no longer identifiable on magnetic resonance imaging.

\section{Results}

The table summarises the radiological, echocardiographic, and operative findings in all our patients.

In all patients with a complete left sided pericardial defect axial MR tomography in supine position showed that the mobile heart had completely fallen into the left hemithorax (fig 4). Thus the left atrium was no longer preaortic but stretched around the descending aorta (figs 2 and 4). Lung tissue interposed between the aorta and the main pulmonary artery replaced the preaortic recess of the pericardial sac (fig 3). The pulmonary artery and the left atrial appendage clearly extended beyond the margins of the normal mediastinum, especially in case 2 (figs 2 and 3). The bulging of the left atrial appendage and the side by side position of the aorta and part of the left atrium were evident on coronal slices too (fig 2). In addition coronal scans showed an area of signal loss in the triangle between the inferior surface of the heart, the aorta, and the diaphragm caused by interposed lung (fig 2).

In the MR tomograms of the patient with a partial left sided pericardial defect the heart showed no leftward displacement. We noted a striking elevation of the apex of the heart, away from the diaphragm (fig 7). A well defined, deep indentation of the outer margin of the left ventricle was visible, especially on axial images. This pleat was most pronounced on the diaphragmatic surface of the heart and extended cranially on both the posterior and anterior walls (figs 7 and 8). Abutting this sulcus was a collarlike accumulation of pericardial fat. At the level of the big vessels the preaortic recess was preserved.

\section{Discussion}

Congenital pericardial defects are rare. They are either complete or partial, occurring more often on the left than the right side. Bilateral complete defects or absence of a defect near the diaphragm is uncommon. ${ }^{6}$

Patients with complete left sided pericardial defect are either asymptomatic or present with non-specific chest pain. No treatment is needed in patients with this diagnosis. Patients with partial left sided pericardial defect, however, are at risk of possibly symptomatic herniation of the atrial appendage or herniation of the left ventricle, eventually leading to fatal myocardial strangulation. Apical defects need to be detected as soon as possible because the time from the onset of symptoms of apical herniation and strangulation of the left ventricle to death varies from one hour to two days. ${ }^{7-13}$

In cases 1 and 2 the diagnosis of complete left sided pericardial defect was missed in childhood because chest radiographs showed a prominence in the hilar region but no characteristic leftward displacement of the heart. This bulge was identified as a diverticulum of the persistent ductus arteriosus in case 1 and as a dilated and strongly pulsating left atrial appendage in case 2 . Thus we suspected a partial left sided pericardial defect since in published reports protrusion of the left atrial appendage is considered pathognomonic for this defect. ${ }^{1-5}{ }^{14-16}$ In both cases complete left sided pericardial defect was diagnosed only intraoperatively. In echocardiograms of patients with complete left sided pericardial defects Payvandi and Kerber and Nicolosi et al described a right ventricular dilatation and a paradoxical or flat movement of the septum, that was simulated due to the abnormal position and mobility of the heart. ${ }^{17} 18$ In our cases 1 and 2, however, $M$ mode echocardiography gave normal results. In case 3 there was only a flat movement of the septum. In all three cases the right ventricular dimensions were normal, whereas the cross sectional images showed an abnormal transverse position of the heart. The dilatation of the left atrial appendage was most pronounced in case 2 .

Magnetic resonance imaging proved to be helpful in all our cases by showing directly all the criteria allowing differentiation of pericardial defects.

In complete left sided pericardial defect the heart has completely fallen into the left hemithorax in scans taken in the supine position owing to the absence of the supporting pericardial sac (figs 2 and 4). Thus the left atrium is wrapped over the descending aorta (fig 2). The preaortic recess is absent and its place is taken by lung tissue (fig 3 ). In coronal scans the lung interposed between the inferior surface of the heart, the diaphragm, and the descending aorta is particularly well visualised (figure 2). Both in partial left sided pericardial defects at atrial level and in complete defects the pulmonary artery and the left atrial appendage are uncovered by pericardium and thus bulge (figures 2 and 3 ). The presence of this bulge combined with a normal position of the heart in the supine position is thus evidence for the presence of a partial left sided pericardial defect in a more cephalad position. Our findings in complete left sided pericardial defects are in concordance with the few reported cases describing signs for this malformation on computed tomography and magnetic resonance imaging. ${ }^{19} 20-24$ In contrast to plain radiography and echocardiography these imaging techniques allow for a clear depiction of the anatomical relations unimpeded by overlying structures. In both computed tomography and magnetic resonance imaging, however, normal pericardium at the level of the atria and ventricles is seen only in the presence of sufficient epicardial and pericardial fat or fluid collection in the pericardial sac. ${ }^{1925}$ Only the preaortic recess is regularly seen. ${ }^{26}$ The higher soft tissue contrast of magnetic resonance imaging offers a clear advantage over computed tomography, but adequate imaging quality is highly dependent 
on patient cooperation and sufficient electrocardiographic synchronisation of the measurement sequence. Motion induced imaging artefacts will be reduced with the introduction of echo planar imaging, in which the scanners have short acquisition times.

Case 4 describes the rare apical location of a partial left sided pericardial defect. Most authors say that in partial left sided pericardial defects the defect occurs in the hilar region behind the ventrally dislocated phrenic nerve, and they warn about the risk of fatal strangulation of the left ventricle. ${ }^{13514-16} \mathrm{We}$ emphasise, however, that in all lethal strangulations of the left ventricle described so far the partial defect was apical and the apex of the left ventricle was always strangulated; two cases showed additional herniation of the left atrial appendage. ${ }^{7-13}$ Although partial defects confined to the atrium can be symptomatic, no patients have died of them and only one case of strangulation of the left atrial appendage has been reported. ${ }^{27}$ Because of the different clinical impact we therefore have to distinguish between the various localisations of partial left sided pericardial defects.

To our knowledge, seven fatal cases of ventricular herniation through an apical pericardial defect have been published; reports at necropsy in six of them mention a deep crest in the myocardium caused by the thickened, free pericardial rim. ${ }^{8-13}$ In one case the appearance of the heart at necropsy is not described. ${ }^{7}$ Our case 4 and three published reports show that this constriction of the myocardium exists even without strangulation. Bruning reported epicardial, pericardial, and myocardial fibrosis and fibrous thickening of the right coronary artery as clear evidence of a long term myocardial constriction before the fatal strangulation. In two other cases of intraoperatively diagnosed partial left sided pericardial defect a myocardial crease was also noted. ${ }^{28} 29$ Therefore this ring-like constriction of the myocardium at the level of the ventricles seems to be the key feature-that is, pathognomonic for life threatening apical pericardial defect.

In our case 4 this constriction ring was obvious on magnetic resonance images as a circumferential indentation of the myocardium along its diaphragmatic surface and the anterior and posterior wall (figures 7 and 8 ); it was also partially visible in echocardiograms (figure 6). Cineangiography showed in diastole the herniation of the ventricle and the compression of the left anterior descending coronary artery at the level of the free pericardial rim (figures 9 and 10). The diastolic narrowing of the left circumflex artery was previously observed only once by Wolff et al in a 27 year old man. ${ }^{30}$

As in our patient, there is no pathognomonic chest radiographic appearance for the herniation of the left ventricular apex through a partial pericardial defect. ${ }^{11228}$ Mainly the clear demonstration of the myocardial constriction groove by means of magnetic resonance imaging allowed for ascertaining this diagnosis in our patient. Apart from the myocardial indentation, the displacement of epicardial and pericardial fat along the compression site was easily visible on magnetic resonance imaging. These conditions exist long before the ventricular strangulation occurs as had been shown by histological studies. ${ }^{10}$ Thus we believe that magnetic resonance imaging should enable an early diagnosis, possibly in time for successful surgical repair.

Combining the findings in our patients with reports published so far we have five main conclusions.

(1) Magnetic resonance imaging with its multiplanar capability allows criteria for both a secure diagnosis and differentiation of left sided pericardial defects to be assessed in one examination.

(2) The complete laterodorsal displacement of the heart into the left hemithorax in supine position of the patient is pathognomonic for complete left sided pericardial defect.

(3) Contrary to previous reports the noticeable prominence of the left atrial appendage cannot be taken as a reliable sign of a partial left sided pericardial defect. It can be seen in both complete and partial left sided pericardial defects.

(4) The bulging of the left atrial appendage with absence of gross cardiac displacement is indicative of a partial left sided pericardial defect atrially. No fatal outcome has been reported in these defects, in which strangulation of the left atrial appendage can occur.

(5) A life threatening ventricular herniation may occur in a partial defect in apical location. The associated myocardial crease of ventricular herniation is pathognomonic and can be seen with modern cross sectional imaging techniques, especially magnetic resonance imaging.

1 Ellis $\mathrm{K}$, Leeds NE, Himmelstein A. Congenital deficiencies in the parietal pericardium. AfR Am $\mathcal{f}$ Roentgenol 1959;82:125-37.

2 Glover LB, Barcia A, Reeves TJ. Congenital absence of the pericardium. AfR Am $\Im$ Roentgenol 1969;106:542-9.

3 Nasser WK, Helmen C, Tavel ME, Feigenbaum H, Fisch C. Congenital absence of the left pericardium. Clinical, electrocardiographic, radiographic, hemodynamic, and angiographic findings in six cases. Circulation 1970;41: 469-78.

4 Pernot C, Hoeffel JC, Henry M, Frisch R, Brauer B Partial left pericardial defect with herniation of the left atrial appendage. Thorax 1972;27:246-50.

5 von Bernuth G, Nissen H, Lang D, Hofstetter R. Kongenitale Defekte des linksseitigen Perikards. $Z$ Kardiol 1976;65:1022-32.

6 Chaurasia BD. Congenital pericardial defects. Teratology 1973;8:55-68.

7 Boxall R. Incomplete pericardial sac; escape of heart into left pleural cavity. Transactions of the Obstetric Society 1887;28:209-10.

8 Sunderland S, Wright-Smith RJ. Congenital pericardial defects. Br Heart $\mathcal{F} 1944 ; 6: 167-75$.

9 Hort W. Hämorrhagische Infarzierung des Herzens bei angeborenem Herzbeuteldefekt. Zentralblatt für Allgemeine Pathologie und Pathologische Anatomie 1962; 103:392-9.

10 Bruning EGH. Congenital defect of the pericardium. f Clin Pathol 1962;15:133-5.

11 Dadds V, Masters PL. Strangulation of the left ventricle following herniation through a congenital defect in the following herniation through a congenital defect in the
pericardial sac. Australian Paediatric foumal 1972;8: pericardial

12 Saito R, Hotta F. Congenital pericardial defect associated with cardiac incarceration: case report. Am Heart $\mathcal{f}$ 1980;100:866-70

13 Jones JW, McManus BM. Fatal cardiac strangulation by congenital partial pericardial defect. Am Heart $\mathcal{f} 1984$; 107:183-5. 
14 Hering AC, Wilson JS, Ball RE. Congenital deficiency of the pericardium. I Thorac Cardiovasc Surg 1960;40: $49-55$.

15 Rogge JD, Mishkin ME, Genovese PD. Congenital partial pericardial defect with herniation of the left atrial appendage. Ann Intern Med 1966;64:137-41.

16 Rowland ThW, Twible EA, Norwood WI, Keane JF. Partial absence of the left pericardium. Am $\mathcal{f}$ Dis Child 1982;136:628-30.

17 Payvandi MN, Kerber RE. Echocardiography in congenital and acquired absence of the pericardium. Circulation 1976;53:86-92.

18 Nicolosi GL, Borgioni L, Alberti E, et al. M-mode and two-dimensional echocardiography in congenital absence of the pericardium. Chest 1982;81:610-3.

19 Moncada R, Baker M, Salinas M, et al. Diagnostic role of computed tomography in pericardial heart disease: congenital defects, thickening, neoplasms, and effusions. gm Heart $71982 ; 103: 263-82$.

20 Baim RS, MacDonald IL, Wise DJ, Lenkei SC. Computed tomography of absent left pericardium. Radiology 1980; 135:127-8.

21 Salem DN, Hymanson AS, Isner JM, Bankoff MS Konstam MA. Congenital pericardial defect diagnosed by computed tomography. Cathet Cardiovasc Diagn 1985;11:75-9.

22 Gutierrez FR, Shackelford GD, McKnight RC, Levitt RG Hartmann A. Diagnosis of congenital absence of left pericardium by MR imaging. $\mathcal{f}$ Comput Assist Tomogr 1985;9:551-3.

23 Schiavone WA, O'Donnel JK. Congenital absence of the left portion of parietal pericardium demonstrated by nuclear magnetic resonance imaging. Am $₹$ Cardiol 1985; 55:1439-40.

24 Millaire A, Goullard L, Tison E, Remy-Jardin M, Santre C, Ducloux G. L'agénesie unilaterale gauche du pericarde. Apport de l'imagérie cardiaque. Arch Mal Coeur 1990;83:275-80.

25 Sechtem U, Tscholakoff D, Higgins CB. MRI of the normal pericardium. AfR Am $\mathcal{f}$ Roentgenol 1986;147: 239-44.

26 McMurdo KK, Webb WR, von Schulthess GK, Gamsu G. Magnetic resonance imaging of the superior pericardial recesses. AfR Am f Roentgenol 1985;145:985-8.

27 Robin E, Ganguly SN, Fowler MS. Strangulation of the left atrial appendage through a congenital partial pericarleft atrial appendage through a cong.

28 Lajos TZ, Bunnell IL, Colokathis BP, Schimert G. Coronary artery insufficiency secondary to congenital pericardial defect. Chest 1970;58:73-6.

29 Wallace HW, Shen D, Baum S, Blakemore WS, Zinsser HF. Angina pectoris associated with a pericardial defect. F Thorac Cardiovasc Surg 1971;61:461-5.

30 Wolff F, Fritz A, Dumeny P, Eisenmann B. Effacement diastolique coronaire dans l'agénésie péricardique gauche partielle. Arch Mal Coeur 1987;2:206-10. 